\title{
Intraoperative Frozen Section Assessment of Sentinel Lymph Nodes in Breast Cancer: Six-Year Experience in a Tertiary Hospital*
}

\author{
Maria Kariza Tolentino-Molina, Sarah Jane Datay-Lim, Elizabeth Ann Alcazaren \\ Section of Anatomic Pathology, Department of Laboratories, The Medical City, Ortigas Avenue, Pasig City
}

\section{ABSTRACT}

Introduction. To determine the reliability of intraoperative frozen section (FS) assessment of sentinel lymph nodes (SLN) in breast cancer patients and describe the factors affecting its evaluation.

Methodology. Records of 245 breast cancer patients with FS of SLNs from December 2007 to December 2013 were retrieved and analyzed. The effect of discordant FS examination and pathology findings on axillary lymph node (ALND) dissection was then evaluated.

Results. Of the total 616 SLNs evaluated, 85 (13.80\%) SLNs were positive on FS, with the majority having a histological diagnosis of invasive ductal carcinoma of no special type (62.04\%). Overall identification rate was $98.36 \%$. Frozen section biopsies had good correlation with permanent sections, with a sensitivity (Sn) of $92.39 \%$, specificity (Sp) of $100 \%$, and a positive predictive value (PPV) of 100\%. Negative cases on FS but were found positive on permanent sections were all cases of micrometastases, giving a false negative rate of $1.31 \%$ and negative predictive value (NPV) of $98.68 \%$. Validation with ALND showed Sn of $100 \%$, Sp of $50 \%$, NPV of $100 \%$, and PPV of $37.17 \%$.

Conclusion. The 6-year data on intraoperative FS reliably evaluated the SLN status of breast cancer patients with a negligible false negative rate. Factors affecting its effectiveness include the predictors of nodal involvement, multilevel sectioning, and size of metastases.

Key words: sentinel node biopsy, breast cancer, axillary lymph node dissection

\section{ISSN 0118-3265}

Printed in the Philippines.

Copyright (C) 2016 by the PJP.

Received: 15 March 2016.

Accepted: 22 April 2016.

Published online first: 27 April 2016.

http://dx.doi.org/10.21141/PJP.2016.006

Corresponding author: Maria Kariza Tolentino-Molina, MD

E-mail:mkltolentino.molina@gmail.com

* This paper was presented in a scientific forum entitled, "Ioint Conference of the Philippine Breast Cancer Society and University of Sto. Tomas Hospital Benavides Cancer Institute," at the University of Santo Tomas, Manila, Philippines, held on October 22-24, 2014.

\section{INTRODUCTION}

With the advent of sentinel lymph node biopsy (SLNB) by frozen section (FS), trends in breast cancer surgery have shifted towards breast-conserving treatment and avoidance of axillary lymph node dissection (ALND) for better quality of life outcomes. In principle, injection of a radioactive isotope and vital blue dye around the area of the tumor allows localization of the first node to receive lymphatic flow which, in principle, is the sentinel lymph node (SLN). The node is then biopsied and examined by routine histopathologic techniques and evaluated for metastasis. If the node is free of metastasis, then it is likely that locoregional spread has not occurred and further ALND is avoided.

The practice of SLNB has been extensively studied since its introduction into clinical practice in the 1990's. The procedure is an extremely sensitive and specific method for predicting whether metastasis has occurred in regional lymph nodes. ${ }^{2}$ The sensitivity of intraoperative FS in identifying nodal metastases within SLNs has been reported to vary within the range of $44 \%$ to $95 \%$, with most studies reporting the sensitivity to be between $60-75 \%$. Advances in histopathologic methods for SLNs allow safe and accurate identification of early breast cancer without axillary node involvement, and SLN is now widely accepted and recommended by the American Society of Clinical Oncology (ASCO) ${ }^{4}$ as it has shown greater benefit in reducing post-operative morbidity and complications like lymphedema, pain, numbness, and limited shoulder movement, which translates to better quality of life (QoL) outcomes. ${ }^{5}$ In line with this, numerous studies have emerged in recent years validating its said advantages. The first large prospective randomized control trial, the Axillary Lymphatic Mapping 
Against Nodal Axillary Clearance (ALMANAC) trial, compared both procedures for comprehensive and repeated quality of life assessments over 18 months. ${ }^{6}$ Similar observations and conclusions on patient outcomes were reported in the National Surgical Adjuvant Breast and Bowel Project (NSABP). ${ }^{6-10}$

Contemporary practice in our setting has since been influenced by promising clinical data. However, studies on the effectiveness of intraoperative FS with SLNB in our country remain few. Here we report the cumulative six year experience at The Medical City and evaluated the sensitivity, specificity, and positive and negative predictive values of intraoperative FS of SLNs. Furthermore, we analysed the impact of discordant FS examination and pathology findings on axillary lymph node dissection.

\section{METHODOLOGY}

Sample size was computed using the OpenEpi open source calculator using the equation: Sample size $n=\left[\mathrm{DEFF}^{*} \mathrm{~Np}(1-\mathrm{p})\right] /$ $\left[\left(\mathrm{d}^{2} / \mathrm{Z}_{1-\alpha / 2}^{2}{ }^{*}(\mathrm{~N}-1)+\mathrm{p}^{*}(1-\mathrm{p})\right]\right.$. Computed Sample size at $95 \%$ confidence level is 237 . Statistical power was likewise determined by open source calculator using the determined sample size of 237 and Alpha error level of $5 \%$ with a resulting statistical power of $45.8 \%$.

Between December 2007 to December 2013, a total of 616 sentinel lymph node biopsies (SLNB) were performed at our institution with the following inclusion criteria: a) Clinical Stage 1, 2A or 2B histologically confirmed Invasive Breast Carcinoma with clinically negative axillary lymph node (ALN); and b) Ductal carcinoma in situ (DCIS) requiring Mastectomy. Patients, likewise, did not have prior chemotherapy or hormonal therapy. On the other hand, exclusion criteria or contraindications were: a) Clinical Stage 3 or 4 Invasive Breast Carcinoma; b) Fine needle aspiration biopsy (FNAB) confirmed ALN positive for metastasis; and c) Women who have undergone extensive breast surgery such as breast reduction or augmentation, as well as extensive axillary surgery such as excision of axillary tumors. SLN was identified using vital blue dye and gamma probe methods as per protocol and submitted for histopathologic examination. All lymph nodes were subjected to FS wherein imprints and tissue sections were taken for examination and intraoperatively reported as either positive or negative for tumor metastasis. Subspecialties (Nuclear Medicine, Surgery, and Pathology) involved in the study followed a protocol which was agreed upon by a concensus within each department. This study was granted an approval from our Institutional Review Board in accordance with the guidelines of the International Conference on Harmonization of Good Clinical Practice (ICH-GCP).

\section{Validation}

Validation of sentinel node status was done by comparing the number of SLN positives and negatives with ALND outcomes. Starting from January 2011, twenty eight (28) of these cases were identified to have a scheduled ALND in spite of a negative SLNB, as indicated in their histopathology forms. These were done upon agreement with their surgeon.

The remaining tissue samples, including those submitted for FS, were subsequently processed on paraffin section. One four (4) micrometer thick section for each lymph node was mounted on a single glass slide. Three such sections (levels) for each lymph node were taken, with an average distance of 40-50 micrometers apartcorresponding to three (3) levels, and stained using hematoxylin and eosin stain (H\&E).

\section{Patients}

Population age ranged from 30 to 81 years old with a mean of 55.5 years, predominantly female gender 243/245 (99.19\%). Most patients were histologically diagnosed as Invasive Ductal Carcinoma 152/245 (62.04\%). The other histologic types: Ductal Carcinoma in situ 27/245 (11.02\%), Invasive Lobular Carcinoma 4/245 (1.63\%) and others (Mucinous, Metaplastic, Apocrine, Invasive Cribriform and Tubular carcinoma) were identified as well 62/245 (25.30\%). One hundred sixty six (166) patients were staged as T1 (7.76\%) and seventy two (72) as T2 (29.39\%), which were the most frequent tumor stages. Lymphovascular space invasion is noted in 60 patients (24.49\%).Tumor biomarker status was also recorded based on the Estrogen receptor (ER), Progesterone receptor (PR), and Human epidermal growth factor receptor2 (HER2) immunohistochemical (IHC) results. Details of patient characteristics are listed on Table 1.

\begin{tabular}{|c|c|}
\hline Variable & Frequency \\
\hline \multicolumn{2}{|l|}{ Age (years) } \\
\hline Range & 31 to 80 \\
\hline Median & 53 \\
\hline Mean & 53.8 \\
\hline \multicolumn{2}{|l|}{ Sex } \\
\hline Male & $2 \quad(0.81 \%)$ \\
\hline Female & 243 (99.19\%) \\
\hline \multicolumn{2}{|l|}{ LVSI } \\
\hline Yes & 60 (24.89\%) \\
\hline No & $185(75.11 \%)$ \\
\hline \multicolumn{2}{|l|}{ HISTOLOGIC TYPE } \\
\hline Invasive Ductal Carcinoma, NST & $152(62.04 \%)$ \\
\hline Ductal carcinoma In situ & $27(11.02 \%)$ \\
\hline Mucinous Carcinoma & $8 \quad(3.27 \%)$ \\
\hline Invasive Lobular Carcinoma & $4 \quad(1.63 \%)$ \\
\hline Metaplastic Carcinoma & $3 \quad(1.22 \%)$ \\
\hline Apocrine Carcinoma & $2 \quad(0.82 \%)$ \\
\hline Invasive Cribriform Carcinoma & $1 \quad(0.41 \%)$ \\
\hline Tubular carcinoma & $1 \quad(0.41 \%)$ \\
\hline Other types & 47 (19.18\%) \\
\hline \multicolumn{2}{|l|}{ Tumor Size* } \\
\hline Tis & $12(11.88 \%)$ \\
\hline T1mic & $4 \quad(3.96 \%)$ \\
\hline T1a & 9 (8.91\%) \\
\hline T1b & $6 \quad(5.94 \%)$ \\
\hline T1c & 29 (28.71\%) \\
\hline $\mathrm{T} 2$ & $28(27.72 \%)$ \\
\hline T3 & $4 \quad(3.96 \%)$ \\
\hline Unknown & 9 (9.91\%) \\
\hline \multicolumn{2}{|l|}{ Estrogen Receptor } \\
\hline Positive & 162 (66.39\%) \\
\hline Negative & 32 (13.11\%) \\
\hline No data & 50 (20.49\%) \\
\hline \multicolumn{2}{|l|}{ Progesterone Receptor } \\
\hline Positive & 146 (59.84\%) \\
\hline Negative & $48 \quad(19.67 \%)$ \\
\hline No data & $50 \quad(20.49 \%)$ \\
\hline \multicolumn{2}{|l|}{ HER2 } \\
\hline Positive & $68 \quad(27.87 \%)$ \\
\hline Negative & 76 (31.15\%) \\
\hline Equivocal & $26(10.66 \%)$ \\
\hline Unknown & $65(26.64 \%)$ \\
\hline
\end{tabular}

\section{RESULTS}

Sentinel lymph nodes (SLN) were successfully identified in 240 of 245 patients with the use of both blue dye and radiolabeled gamma probe, with an identification rate of $98.36 \%$. Lymph nodes submitted for FS ranged from 1 to 13 with an average of 2.6 lymph nodes per examination. This totalled to 616 SLN submitted for FS and subsequent routine paraffin sections; the majority of cases were negative on FS (531 SLN or 86.20\%) with 524 true negatives on paraffin sections. All positive SLNs on FS were likewise positive on 

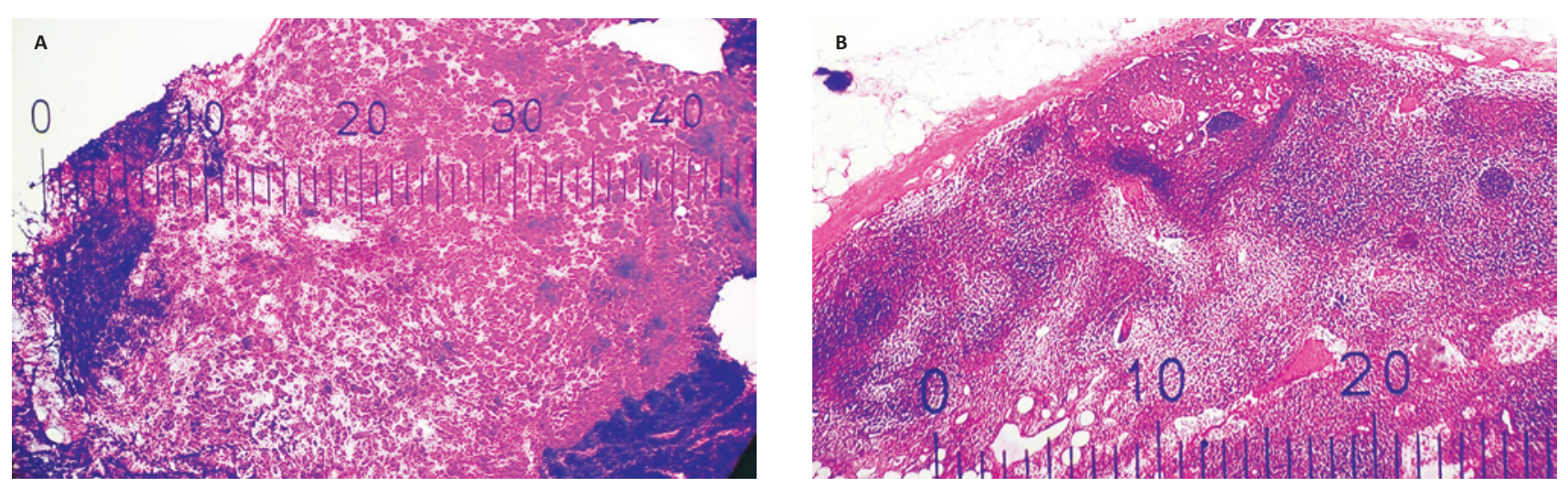

Figure 1. Sentinel lymph nodes with macrometastasis and micrometastasis. (A) Photomicrograph of a macrometastasis (>2 $\mathrm{mm})$ in a sentinel lymph node (100x, H\&E); (B) Photomicrograph of a micrometastasis (<2 mm) in a sentinel lymph node (400x, H\&E).

paraffin sections (85 TP, 0 FP). These results yielded a sensitivity of $92.39 \%$ (CI 84.94 - 96.97) and a specificity of $100 \%$ (CI 99.29 100). Our study shows that intraoperative FS has a $98.68 \%$ negative predictive value when confirmed with subsequent paraffin sections (See Table 2).

Consequently, these results were validated by eighty one cases that underwent subsequent ALND, wherein 53 cases had positive SLNs and 28 cases had negative SLNs. Out of the 53 positive SLNs, $50.1 \%$ or 27 cases were found to be negative for metastasis on subsequent ALND, while $49.16 \%$ or 26 cases were found to be positive on subsequent ALND. Out of the 28 cases with negative SLNs, all or $100 \%$ were confirmed to be negative on subsequent ALND. There were no false negatives by ALND out of the 81 cases observed in this subset. This corresponds to $100 \%$ sensitivity, $50 \%$ specificity, $100 \% \mathrm{NPV}$, and $47.17 \%$ PPV of intraoperative FS when compared to ALND results.

The tumor deposits were evaluated upon routine paraffin examination and categorized either as micrometastases $(<2.0 \mathrm{~mm})$ or macrometastases $(>2.0 \mathrm{~mm}$ ) (See Figure 1). FS of SLN detected mostly macrometastases in $67(78.82 \%)$ of the 85 positive cases. Micrometastases were also detected in 18 cases (21.18\%). The ALN dissection performed on the 81 patients with positive SLN yielded 53 patients (65.43\%) confirmed positive on ALND, while 28 patients $(34.67 \%)$ were negative. Eighty two (82) of the SLN positive patients were histologically diagnosed as Invasive Ductal Carcinoma, while 6 were classified as Ductal Carcinoma in situ. Breast cancers staged as T2 comprised $27.72 \%$ of the cases, with LVSI noted in $24.89 \%$ (See Table 3).

\section{DISCUSSION}

Currently, our centre has an overall identification rate for SLN biopsy at $98.36 \%$.Validation of sentinel node status was done by comparing the number of SLN positive and negatives with ALND outcomes. Our results demonstrate that out of the 53 positive SLNs, 50.1\% or 27 cases were found to be negative for metastasis on subsequent ALND, and out of 28 negative SLNs, $100 \%$ were confirmed negative in ALND. This finding support the concept of sentinel lymph node as the first lymph node or group of nodes encountered in the lymphatic drainage of the breast. Aside from this, it validates that the technique done by the surgeons in identification of sentinel node is acceptable because all the negative sentinel nodes were indeed negative on the subsequent axillary node dissection.
It can be concluded from our results that proper technique and meticulous screening with intraoperative FS of SLNB reliably identifies locoregional metastasis. Identification of these metastasispositive nodes through SLN technique allows the surgical practitioner to harvest positive nodes only and avoid aggressive ALND. Also important to note, given the high sensitivity for negative SLNs, subsequent ALND may not be performed which may spare the patient from other morbidities.

According to studies, variables affecting the procedure are the following: age, pathological tumor size, histology, year of accrual, and method of detection. ${ }^{11}$ Predictors of further nodal involvement are tumor size, lymphovascular space invasion (LVSI) and lobular histology. ${ }^{12}$

A positive FS can save the patient a second reoperation for completion axillary lymph node dissection and a negative FS

Table 2. 616 Sentinel lymph nodes submitted for frozen section

\begin{tabular}{llll}
\hline & Positive & Negative & \\
\hline Positive & TP 85 & FP 0 & PPV 100\% \\
Negative & FN 7 & TN 524 & NPV 98.68\% \\
& Sensitivity 92.39\% & Specificity 100\% & \\
\hline
\end{tabular}

Table 3. Descriptive characteristics of sentinel lymph node

\begin{tabular}{|c|c|}
\hline Variable & Frequency \\
\hline Total Number of submitted SLN for 245 patients & 616 \\
\hline Range & $1-19$ \\
\hline \multicolumn{2}{|l|}{ Mean } \\
\hline No. of Positive SLN & $85(13.80 \%)$ \\
\hline Micrometastasis $(<2.0 \mathrm{~mm})$ & $9 \quad(10.59 \%)$ \\
\hline Macrometastasis (>2.0mm) & 27 (31.76\%) \\
\hline No. of Negative SLN & $531(86.20 \%)$ \\
\hline Validation by Axillary Dissection & 81 \\
\hline SLN Positive Patients with Axillary Dissection & 25 \\
\hline Positive ALN & $26(32.5 \%)$ \\
\hline Negative ALN & 54 (42.86\%) \\
\hline \multicolumn{2}{|l|}{ LVSI } \\
\hline Present & $20(80.0 \%)$ \\
\hline Absent & $5(20.0 \%)$ \\
\hline \multicolumn{2}{|l|}{ Tumor Deposit Size (in $\mathrm{mm}$ ) } \\
\hline Range & $0.1-26$ \\
\hline Mean & 6.05 \\
\hline \multicolumn{2}{|l|}{ Tumor size of Positive SLN cases } \\
\hline T1a & $1 \quad(1.80 \%)$ \\
\hline T1b & $1 \quad(1.80 \%)$ \\
\hline T1c & $15(26.79 \%)$ \\
\hline $\mathrm{T} 2$ & $28 \quad(50.00 \%)$ \\
\hline T3 & $4 \quad(7.14 \%)$ \\
\hline
\end{tabular}

Note: SLN = Sentinel Lymph Node; ALN = Axillary Lymph Node LVSI = Lymphovascular space invasion 
can spare the patient from ALND completely, avoiding all the associated morbidities. This puts a lot of pressure on the decision making done during intraoperative FS because it can adversely affect outcome and influence management. Our data showed that there is good correlation between intraoperative FS and permanent paraffin H\&E sections with a sensitivity of $92.39 \%$, and specificity and positive predictive value of $100 \%$.

Discordant FS results were noted on seven SLN negative cases (8.23\%) where micrometastases were noted only on permanent sections $(7 / 85)$. The false negative rate was $1.31 \%$ and NPV was $98.68 \%$. Those were observed during the early course of introduction of this method at our hospital. As we gained experience, multiple levels (2) or step sections were done on each of the submitted SLN for FS, which eliminated our false negative results since October of 2010. Still, other studies have reported higher false negativity at $11 \%{ }^{2}$ and discordance rate of FS at $17 \%{ }^{13}$ Therefore, limitations inherent to the procedure should always be taken into consideration. Frozen section may fail to detect micrometastases. Apart from doing multilevels on FS, immunohistochemistry (IHC), particularly antibodies to cytokeratin have improved the identification of SLN. Even though this technique was not included in our protocol, studies have shown that IHC has been reported to upstage the disease in approximately $10 \%$ of patients with negative SLN. ${ }^{2}$ This can improve identification of micrometastasis and isolated tumor cells that maybe difficult to identify even with routine $\mathrm{H} \& \mathrm{E}$ technique.

Submitted SLN for FS range from 1 to 13 lymph nodes, with an average of 2. A study concluded that this may either be due to the migration of dye or isotope from the true SLN to secondary lymph nodes or a normal anatomic variation in which the lymphatics of a given site in the breast drain simultaneously. ${ }^{2}$

\section{CONCLUSION}

Intraoperative FS can reliably evaluate the SLN status of women with early breast cancer but it may fail to detect micrometastases. Factors affecting the effectiveness of intraoperative FS of SLN include the predictors of nodal involvement (size of tumor, histology, lymphovascular space invasion), number of step sections, and size of metastases.

\section{AUTHOR DISCLOSURE}

The authors declared no conflicts of interest.

\section{REFERENCES}

1. Belmonte R, Garin O, Segura M, et al. Quality-of-life impact of sentinel lymph node biopsy versus axillary lymph node dissection in breast cancer patients. Value Health. 2012;15(6):907-15. http://dx.doi.org/10.1016/j.jval.2012.06.003.

2. Carvalho SMT, Netto MM, Lima ENP, et al. Sentinel node biopsy in breast cancer: results in a large series, Braz J Med Biol Res. 2010;43(6):593-9.http://dx.doi.org/10.1590/S0100879X2010007500048.
3. Moatasim A, Mujtaba S, Faridi N. Intraoperative frozen section analysis of sentinel lymph nodes in breast carcinoma patients in a tertiary hospital in Pakistan. Int J Surg. 2013;11(3):253-8. http://dx.doi.org/10.1016/j.jjsu.2013.01.007.

4. Lyman GH, Temin S, Edge SB, et al. Sentinel lymph node biopsy for patients with early-stage breast cancer: American Society of Clinical Oncology Clinical Practice Guideline Update. J Clin Oncol. 2014;32(13):1365-83. http://dx.doi. org/10.1200/JCO.2013.54.1177.

5. Velloso FS, Barra AA, Dias RC. Functional performance of upper limb and quality of life after sentinel lymph node biopsy of breast cancer. Braz J Phys Ther. 2011;15(2):146-53.

6. Fleissig A, Fallowfield LJ, Langridge CI, et al. Post-operative arm morbidity and quality of life. Results of the ALMANAC randomised trial comparing sentinel lymph node biopsy with standard axillary treatment in the management of patients with early breast cancer. Breast Cancer Res Treat. 2006;95(3):279-3. http://dx.doi.org/10.1007/s10549-005-9025-7.

7. Land SR, Kopec JA, Julian TB, et al. Patient-reported outcomes in sentinel node-negative adjuvant breast cancer patients receiving sentinel node biopsy or axillary dissection: National Surgical Adjuvant Breast and Bowel Project Phase III Protocol B-32. J Clin Oncol. 2010;28(25):3929-36. http://dx.doi.org/10.1200/JCO.2010.28.2491.

8. Zavagno G, De Salvo GL, Scalco G, et al. A randomized clinical trial on sentinel lymph node biopsy versus axillary lymph node dissection in breast cancer: results of the Sentinella/ GIVOM Trial. Ann Surg. 2008;247(2):207-13. http://dx.doi. org/10.1097/SLA.0b013e31812e6a73. PMID: 18216523.

9. Tice JA. Sentinel lymph node biopsy (SNLB) in early stage breast cancer. California technology assessment forum, California: San Francisco, pp.1-40, Oct. 2011.

10. Kootstra JJ, Hoekstra-Weebers JEHM, Rietmann JS, et.al. A longitudinal comparison of arm morbidity in stage I-II breast cancer patients treated with sentinel lymph node biopsy, sentinel lymph node biopsy followed by completion lymph node dissection, or axillary lymph node dissection. Ann Surg Oncol. 2010; 17(9):2384-94. http://dx.doi.org/10.1245/ s10434-010-0981-8.

11. Straver ME, Meijnen P, van Tienhoven G, et al. Sentinel node identification rate and nodal involvement in the EORTC 10981-22023 AMAROS trial. Ann Surg Oncol. 2010;17(7):1854-61. http://dx.doi.org/10.1245/s10434-0100945-z.

12. Postaci H, Zengel B, et al. Sentinel lymph node biopsy in breast cancer: predictors of axillary and non-sentinel lymph node involvement. Balkan Med J. 2013;30:415-21. http:// dx.doi.org/10.5152/balkanmedj.2013.9591.

13. Geertsema D, Gobardhan PD, Madsen EVE, et al. Discordance of intraoperative frozen section analysis with definitive histology of sentinel lymph nodes in breast cancer surgery: complementary axillary lymph node dissection is irrelevant for subsequent systemic therapy. Ann Surg Oncol. 2010;17(10):2690-5. http://dx.doi.org/10.1245/s10434-010-1052-x.

\footnotetext{
Disclaimer: This journal is OPEN ACCESS, providing immediate access to its content on the principle that making research freely available to the public supports a greater global exchange of knowledge. As a requirement for submission to the PJP, all authors have accomplished an AUTHOR FORM, which declares that the ICMJE criteria for authorship have been met by each author listed, that the article represents original material, has not been published, accepted for publication in other journals, or concurrently submitted to other journals, and that all funding and conflicts of interest have been declared. Consent forms have been secured for the publication of information about patients or cases; otherwise, authors have declared that all means have been exhausted for securing consent.
} 